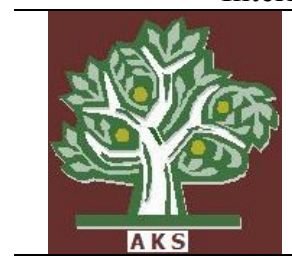

Internet Journal of Medical Update

\author{
Journal home page: http://www.akspublication.com/ijmu
}

\title{
Editorial
}

\section{Port-site infections due to non-tuberculous mycobacteria (atypical mycobacteria) in laparoscopic surgery}

\author{
Vipul D Yagnik
}

\section{Consultant endoscopic and laparoscopic surgeon, Ronak Endo-laparoscopy and General Surgical Hospital, Patan, Gujarat, India}

Acid-fast mycobacteria that do not produce tuberculosis or Hansen's disease are grouped under non-tuberculous mycobacteria (NTM) or atypical mycobacteria ${ }^{1}$. They exist in soil, water, and animal sources. Most human infections, including port-site infections, are acquired from the environment or external sources. The number of cases has increased recently because of improved diagnostic techniques such as culture and increase in the number of immunocompromised hosts. ${ }^{1}$ Laparoscopic surgery has brought about a paradigm shift in surgical technique. Nowadays, most surgeries can be performed via laparoscopic or minimal access surgery. Port-site infections, particularly those associated with NTM, have been a source of significant morbidity in the patients operated with a laparoscopic procedure. The NTM can colonize in tap water, natural water, sewage, and soil, thereby easily infecting solutions and disinfectants used in hospitals. ${ }^{2}$ Most surgeries performed through laparoscopy leave class 1 and 2 wounds. Immunosuppression due to a breach in the continuity of the skin or any disease can lead to infections with patients' own flora.

Mycobacterium fortuitum, $M$. chelonae, $M$. abscessus, M. flavescens, and M. massiliense are commonly associated with NTM port-site infections. Rapidly growing mycobacteria produce mature growth on a media plate within 7 days $^{\mathbf{3}}$. Among the rapidly growing non-tuberculous mycobacteria, M. fortuitum, M. chelonae, and $M$. abscessus are the most common. M. fortuitum is responsible for $60 \%$ of the localized infections in previously healthy individuals. $M$. chelonae is seen primarily in patients who are immunocompromised. Meanwhile, M. abscessus is not as common but may also cause infection in both healthy and immunocompromised individuals ${ }^{3}$.

They have a predilection for the skin and subcutaneous tissue, and cause port-site infections 3 - 4 weeks post-surgery. Non-tuberculous or atypical mycobacterial port-site infection has five clinical stages. ${ }^{2}$
Stage 1: A small tender nodule in the vicinity of the port site.

Stage 2: Increase in the size and tenderness with a sign of inflammation, a nodule, followed by discharge of white pus.

Stage 3: Reduced pain with continuously discharging sinus and necrosis of the overlying skin.

Stage 4: Chronic sinus with white or serous discharge.

Stage 5: Hyperpigmentation with necrosed skin and appearance of nodules at the other site.

Durate et $a l^{4}$ observed that the following factors may be responsible for NTM infections: spread in aquatic environments for a long time, inadequate mechanical cleaning of surgical instruments, or dissemination inside commercially available nonactivated glutaraldehyde solutions. Some surgical teams carry their instruments and perform surgeries at different hospitals and even different cities and states. Some reuse disposable or improperly sterilized trocars.

Earle H. Spaulding devised a rational approach to sterilization of patients care items or instruments. The three categories Spaulding described were: critical, semi-critical and noncritical. Most laparoscopic equipment comes under critical items and requires sterilization. Laparoscopes, arthroscopes, and other scopes that normally enter sterile tissue should be sterilized before each use; if this is not feasible, they should receive at least high-level disinfection ${ }^{5}$. An item should be rinsed with sterile water to prevent its contamination with non-tuberculous mycobacteria.

For laparoscopy, the standard procedure is to sterilize the instrument with $2-2.5 \%$ glutaraldehyde for 20 minutes. It acts as a disinfectant, not a sterilizing agent, so spores often survive. The contaminated instrument deposits the endospore in the subcutaneous tissue during the laparoscopic procedure, which then germinates, followed by the appearance of symptoms after an incubation period of 3-4 weeks. 
A study by Lorena et $a l^{6}$ on the $M$. massiliense BRA100 strain showed that it is resistant to even a higher concentration of glutaraldehyde (GTA, 7\%). Hence, they proved that glutaraldehyde might not be effective for rapidly growing mycobacteria. Other liquid sterilizing agents such as orthophthaldehyde (OPA; $0.55 \%$ ) and peracetic acid may be substituted instead of glutaraldehyde for high-level disinfection with good efficacy. ${ }^{5}$ The contact time required for OPA to take effect is 12 minutes, at which time it destroys all bacteria, fungi, and mycobacteria. Hydrogen peroxide gas plasma and vaporized hydrogen peroxide have been effective in killing NTM. Ethylene oxide (ETO) is also a good alternative for heat-sensitive instruments.

In a majority of cases, the source of infection is direct or indirect contamination of the port site due to colonized water. ${ }^{\mathbf{7 , 8}}$

Diagnosis is established by culture of the wound discharge or tissue biopsy. The material needs to be processed for bacilloscopy, culture in LowensteinJensen medium, and BACTEC technique.' Molecular techniques such as polymerase chain reaction (PCR) and restriction fragment length polymorphism should be used to identify nontuberculous mycobacteria, including $M$. chelonae. ${ }^{\mathbf{1 0}}$ Resistance to polymyxin B disk $(300 \mu \mathrm{g})$ can be the simplest and most accurate method for rapid identification of $M$. chelonae. ${ }^{11}$ A high index of suspicion is required for diagnosis. Increased Creactive protein level with normal total and differential counts may suggest the possibility of atypical mycobacterial infection. Tissue biopsy can reveal chronic granulomatous inflammation. In the case of chronic granulomatous inflammation, enzyme-linked immunospot ${ }^{\mathbf{1 2}}$ and PCR assays (Xpert $\mathrm{MTB} / \mathrm{RIF})^{\mathbf{1 3}}$ are novel, rapid, and noninvasive tests to rule out $M$. tuberculosis infection.

Treatment options have been found to vary depending on the stage of the disease. Small lesions require antibiotics and local wound dressing. Extensive lesions require surgical debridement. ${ }^{\mathbf{1 4}}$

Table 1: Treatment options

\begin{tabular}{|l|l|}
\hline Species & Drugs \\
\hline M. fortuitum & $\begin{array}{l}\text { Amikacin, cefoxitin, ciprofloxacin, } \\
\text { moxifloxacin, clarithromycin, } \\
\text { doxycycline, sulphonamides, } \\
\text { linezolid and imipenem }\end{array}$ \\
\hline M. abscessus & $\begin{array}{l}\text { Amikacin, cefoxitin, } \\
\text { clarithromycin and imipenem }\end{array}$ \\
\hline M. chelonae & $\begin{array}{l}\text { Amikacin, imipenem, } \\
\text { clarithromycin, tobramycin, and } \\
\text { sometimes linezolid }\end{array}$ \\
\hline
\end{tabular}

Cutaneous infections associated with atypical mycobacteria should be treated according to guidelines for treatment for the specific species involved.

Tobramycin is more active than amikacin for $M$. chelonae. Clarithromycin is sufficient for localized skin and soft tissue infection caused by $M$. chelonae and $M$. abscessus. For disseminated disease, 4-6 month therapy is recommended. Most are sensitive to macrolide and aminoglycoside. However, susceptibility testing is recommended. ${ }^{\mathbf{1 5}}$ Macrolide monotherapy for localized infections with $M$. chelonae may be sufficient, whereas multiple antimicrobial drugs are essential if the infection is disseminated or if other species are involved. In localized infections involving isolates of the M. fortuitum group, other agents may be necessary due to the presence of the erm gene, which may induce resistance to macrolides ${ }^{\mathbf{1 6}}$.

Take home message for prevention of nontuberculous mycobacterial infection in the laparoscopic surgery:

- Implementation of strict aseptic protocol.

- Laparoscopes and metallic ports that normally enter sterile tissue should be sterilized before each use; if this is not feasible, they should receive at least high-level disinfection.

- The instruments should be thoroughly cleansed after each procedure, with complete dismantling of parts to ensure removal of all organic soil including blood particles or clots.

- Use of disposable trocars and instruments, and adequate availability of properly sterilized reusable trocars to cover all the surgical procedures in a day.

- Use of autoclave water to rinse the instrument.

- Replace the glutaraldehyde solution with OPA.

- Use ethylene oxide gas sterilization for heat sensitive instruments.

- Hydrogen peroxide gas plasma and vaporized hydrogen peroxide should be utilized if available.

\section{REFERENCES}

1. James WD, Berger TG, Eston DM. Mycobacterial disease. Andrew's Disease of the Skin. 12th edition. Elsevier, Philadelphia. 2016:319-30.

2. Chaudhuri S, Sarkar D, Mukerji R. Diagnosis and management of atypical mycobacterial infection after laparoscopic surgery. Indian $J$ Surg. 2010;72:438-442.

3. Brown-Elliott BA, Wallace Jr RJ. Infections caused by nontuberculous mycobacteria otherthan Mycobacterium avium Complex. In Mandell, Douglas and Bennett's Principles and 
Practice of Infectious Disease, Bennett JE, Dolin R and Blaser (editors). 8th edition, Elsevier, Philadelphia.

4. Duarte RS1, Lourenço MC, Fonseca Lde S, Leão SC, et al. Epidemic of postsurgical infections caused by Mycobacterium massiliense. $J$ Clin Microbiol. 2009;47(7):2149-55.

5. Rutala WA, Weber D, HICPAC. Guideline for disinfection and sterilization in healthcare facilities, 2008. Available from: https://www.cdc.gov/infectioncontrol/guidelin es/disinfection/

6. Lorena NS, Pitombo MB, Côrtes PB, Maya $\mathrm{MC}$, et al. Mycobacterium massiliense BRA100 strain recovered from postsurgical infections: resistance to high concentrations of glutaraldehyde and alternative solutions for high level disinfection. Acta Cir Bras. 2010;25:455-9.

7. Chowbey P. Total extraperitoneal repair of hernia. Endoscopic repair of abdominal wall hernia, 2nd edition. bywordsbooks, delhi 2012:110-30.

8. Vijayaraghavan R, Chandrashekhar R, Sujatha $\mathrm{Y}$, Belagavi CS, et al. Hospital outbreak of atypical mycobacterial infection of port sites after laparoscopic surgery. J Hosp Infect. 2006;64(4):344-7.

9. Sasmal PK, Mishra TS, Rath S, Meher S, et al. Port site infection in laparoscopic surgery: A review of its management. World J Clin Cases. 2015;3(10):864-71.

10. Lee H, Park HJ, Cho SN, Bai GH, et al. Species identification of mycobacteria by
PCR-restriction fragment length polymorphism of the rpoB gene. $J$ Clin Microbiol. 2000;38(8):2966-71.

11. Wallace RJ Jr, Swenson JM, Silcox VA, Good $\mathrm{RC}$, et al. Disk diffusion testing with polymyxin and amikacin for differentiation of Mycobacterium fortuitum and Mycobacterium chelonae. J Clin Microbiol. 1982;16:1003-6.

12. Cho OH, Park KH, Park SJ, Kim SM, et al. Rapid diagnosis of tuberculous peritonitis by $\mathrm{T}$ cell-based assays on peripheral blood and peritoneal fluid mononuclear cells. J Infect. 2011;62(6):462-71.

13. Vadwai V, Boehme C, Nabeta P, Shetty A, et al. Xpert MTB/RIF: a new pillar in diagnosis of extrapulmonary tuberculosis? J Clin Microbiol. 2011;49(7):2540-5.

14. Brown-Elliott BA, Nash KA, Wallace RJ Jr. Antimicrobial susceptibility testing, drug resistance mechanisms, and therapy of infections with nontuberculous mycobacteria. Clin Microbiol Rev. 2012;25:545-82.

15. Akram S, Bhimji S. Mycobacterium Chelonae. StatPearls Publishing; Treasure Island. 2017.

16. Nash KA, Zhang Y, Brown-Elliott BA, Wallace RJ Jr. Molecular basis of intrinsic macrolide resistance in clinical isolates of Mycobacterium fortuitum. $J$ Antimicrob Chemother. 2005;55:170-7

$$
\begin{array}{r}
\text { Vipul Yagnik } \\
\text { Associate Editor } \\
\text { Email: vipulyagnik78@gmail.com }
\end{array}
$$

